\title{
Early exposure to bio-contaminants and asthma up to 10 years of age: results of the HITEA study
}

\author{
Christina Tischer ${ }^{1}$, Lidia Casas ${ }^{2,3,4,5}$, Inge M. Wouters ${ }^{6}$, Gert Doekes ${ }^{6}$, \\ Raquel Garcia-Esteban ${ }^{3,4,5}$, Ulrike Gehring ${ }^{6}$, Anne Hyvärinen?, \\ Marieke Oldenwening ${ }^{6}$, Marjan Kerkhof ${ }^{8}$, Jordi Sunyer ${ }^{3,4,5,9}$, Marie Standl ${ }^{1}$, \\ Elisabeth Thiering ${ }^{1,10}$, Maties Torrent ${ }^{11}$ and Joachim Heinrich ${ }^{1}$ for the HITEA \\ study group
}

\begin{abstract}
Affiliations: ${ }^{1}$ Institute of Epidemiology I, Helmholtz Zentrum München, German Research Centre for Environmental Health, Neuherberg, Germany. ${ }^{2}$ Dept of Public Health and Primary Care - Centre for Environment and Health KU Leuven, Leuven, Belgium. ${ }^{3}$ Centre for Research in Environmental Epidemiology (CREAL), Barcelona, Spain. ${ }^{4}$ CIBER Epidemiología y Salud Pública (CIBERESP), Barcelona, Spain. ${ }^{5}$ Hospital del Mar Medical Research Institute (IMIM), Barcelona, Spain. ${ }^{6}$ Institute for Risk Assessment Sciences, Division Environmental Epidemiology, Utrecht University, Utrecht, The Netherlands. ${ }^{7}$ Dept Environmental Health, National Institute for Health and Welfare, Kuopio, Finland. ${ }^{8}$ University Medical Center Groningen, Dept of Epidemiology, University of Groningen, Groningen, The Netherlands. ${ }^{9}$ University Pompeu Fabra, Barcelona, Spain. ${ }^{10}$ Division of Metabolic Diseases and Nutritional Medicine, Dr von Hauner Children's Hospital, Ludwig-Maximilians-University, Munich, Germany. ${ }^{11}$ Area de Salud de Menorca, IB-SALUT, Mahon, Spain.
\end{abstract}

Correspondence: Christina Tischer, Institute of Epidemiology I, Helmholtz Zentrum München, German Research Centre for Environmental Health, Ingolstaedter Landstrasse 1, D-85764 Neuherberg, Germany.

E-mail: christina.tischerahelmholtz-muenchen.de

ABSTRACT Inverse associations have been found between exposure to bio-contaminants and asthma and allergies. The aim of this study was to prospectively assess whether early exposure to bio-contaminants in dust is associated with asthma and allergy later in childhood among children from (sub)-urban areas.

In subsets of three European birth cohorts (PIAMA: $n=553$; INMA: $n=481$; and LISAplus: $n=395$ ), endotoxin, (1,3,)- $\beta$-D-glucan and extracellular polysaccharide were measured in dust from living rooms shortly after birth. Current asthma at 6 years and 10 years of age and ever asthma up to 10 years of age were assessed by parental questionnaires. Specific IgE levels at 8 years (PIAMA) and 10 years (LISAplus) were available. Adjusted, cohort-specific logistic regression analyses were performed.

Higher endotoxin concentrations were positively associated with current asthma at 6 years of age in PIAMA (adjusted OR 1.96, 95\% CI 1.07-3.58), but were inversely related with ever asthma up to 10 years of age in INMA (adjusted OR 0.39, 95\% CI 0.16-0.94). No associations with asthma were found for LISAplus. No associations were observed with atopic sensitisation in all cohorts. All associations with $(1,3)$ - $\beta$-D-glucan and extracellular polysaccharide were statistically nonsignificant.

The suggested immunological mechanisms of early exposure to bio-contaminants with regards to asthma and allergy might be different for children growing up in (sub)-urban environments.

This article has supplementary material available from erj.ersjournals.com

Received: Oct 312013 | Accepted after revision: Aug 062014 | First published online: Sept 032014

Conflict of interest: None declared.

Copyright OERS 2015 


\section{Introduction}

The perinatal phase is thought to be a crucial time for the development and maturation of the immune system. Environmental microbial exposure during this time may play a major role in the pathogenesis of asthma and allergies [1]. Viable and non-viable microbial agents are ubiquitous in indoor environments, are recognised by the innate immune system, and can cause an inflammatory response [2]. Studies among children living in farming and rural environments have observed that measured levels of microbial agents in settled house dust, such as bacterial endotoxin or (1,3)- $\beta$-D-glucan, were inversely associated with asthma and allergic disorders [3-5]. These observations were confirmed using a prospective approach in the rural PASTURE (Protection against Allergy: Study in Rural Environments) birth cohort study, which showed that bacterial endotoxin and fungal extracellular polysaccharide (EPS) levels measured in floor dust shortly after birth were inversely related to asthma and wheezing without a cold in 2-year-old children [5]. It has also been suggested that the protective effects of microbial exposure may be more pronounced for atopic asthma and in subjects from non-farming households [6, 7].

Previous studies on birth cohorts with participants in (sub)-urban environments that examined similar associations have yielded inconsistent results. Inverse associations have been observed between exposure to higher levels of bio-contaminants in settled dust in the first months of life and nonspecific eczema at 1 year of age [8], recurrent wheezing at 18 months of age [9], asthma at 4 years of age [10] and atopic sensitisation at 7 years of age [11]. Another recent HITEA (Health Effects of Indoor Pollutants: Integrating microbial, toxicological and epidemiological approaches) study by CASAS et al. [12], conducted using the same study subjects as in the present study, reported that early exposure to endotoxin was inversely associated with exhaled nitric oxide at 10 years of age in a combined analysis. In contrast, exposure to higher levels of endotoxin from family room and living room floor dust, measured shortly after birth, was found to increase the risk of repeated and ever wheezing up to the age of 7 years in a US birth cohort based in the metropolitan area of Boston (MA, USA) [11, 13, 14]. A positive association between higher endotoxin levels in mattress dust and repeated wheeze up to 2 years of age has also been observed among children from the German LISAplus study [15].

The effects of early exposure to bio-contaminants in relation to health effects at later ages in childhood remain under studied. Studies with health data covering a wide age range are rare. Birth cohort studies with a long follow-up and conducted in (sub)-urban environments are, in this context, of particular interest, especially when different geographical regions can be simultaneously studied. Therefore, the aim of the present study was to prospectively evaluate whether early exposure to endotoxin, (1,3)- $\beta$-D-glucan and EPS, measured in settled dust from the living room at 3 months of age, are associated with the development of asthma and atopy up to 10 years of age in subsets of three European birth cohorts from (sub)-urban environments.

\section{Methods}

\section{Study population and dust collection}

As part of the European HITEA project, the present study includes information from three ongoing European birth cohorts initiated between 1996 and 1999: the whole INMA-Menorca (INfancia y Medio Ambiente - Childhood and Environment) cohort from Spain [16], and subsamples from both the PIAMA (Prevention and Incidence of Asthma and Mite Allergy) cohort from the Netherlands [17] and the LISAplus (Influence of Life-style factors on the development of the Immune System and Allergies in East and West Germany) cohort from Germany [18]. In PIAMA, microbial levels in house dust were only sampled for children from the intervention arm of the study (children of allergic and/or asthmatic mothers). For LISAplus, a random sample of LISAplus participants with dust samples were included in the HITEA project. Participants in this sub-sample had a high response rate from birth to 10 years of age,

Support statement: This work was supported by the European Commission as part of HITEA (grant agreement no. 211488 under the Seventh Framework Programme, topic ENV.2007.1.2.1.1). The first 2 years of the LISAplus study were mainly supported by grants from the Federal Ministry for Education, Science, Research and Technology, Helmholtz Zentrum Munich (former GSF; Neuherberg, Germany), the Helmholtz Centre for Environmental Research - UFZ (Leipzig, Germany) and the Research Institute at Marien-Hospital Wesel, Paediatric Practice (Bad Honnef, Germany). The 4, 6 and 10-year follow-up examinations of the LISAplus study were covered by the respective budgets of the partners involved (Helmholtz Zentrum Munich (former GSF), Helmholtz Centre for Environmental Research - UFZ, the Research Institute at Marien-Hospital Wesel, Paediatric Practice and the IUF - Leibniz-Research Institute for Environmental Medicine at the University of Düsseldorf (Dusseldorf, Germany)) and by a grant from the Federal Ministry for Environment (IUF Düsseldorf, FKZ 20462296). The PIAMA study is supported by the Netherlands Organization for Health Research and Development (The Hague, The Netherlands), the Netherlands Organization for Scientific Research (The Hague), the Netherlands Asthma Fund (Amersfoort), the Netherlands Ministry of Spatial Planning, Housing, and the Environment (The Hague), and the Netherlands Ministry of Health, Welfare, and Sport (The Hague). The INMA-Menorca study was funded by Fondo de Investigacion Sanitaria, ISCIII, Ministerio de Sanidad y Servicios Sociales (Madrid, Spain; grants 97/0588, 00/0021-2, G03/176, PI061756 and PS0901958), EC Contract QLK4-CT-2000-00263 and Fundacio Roger Torne (Barcelona, Spain). 
were preferably children who had not moved and were from the city of Munich, Germany. Information on how the sub-samples were selected for the HITEA project are detailed elsewhere [19]. Written informed consent was obtained from all parents and the studies were approved by the local ethics committees in each region. The present study includes children with measured endotoxin, (1,3)- $\beta$-D-glucan and EPS levels in living room dust collected shortly after birth (PIAMA: $n=553$; INMA: $n=481$; LISAplus: $n=395$ ). Using vacuum cleaners (LISAplus: Phillips, Hamburg, Germany; PIAMA: Rowenta, Offenbach, Germany; INMA: Globin, Leeds, UK) equipped with ALK filter holders (LISAplus and PIAMA: ALK, Hørsholm, Denmark; INMA: ALK, Maidstone, UK) containing a paper filter, living room dust samples were collected in the homes of the participants when the child was 2-3 months of age. Samples were collected from living room floors in LISAplus and PIAMA, and from living room sofas in INMA. A detailed description of the dust sampling and analysis procedures has been published previously [19].

\section{Health information}

Parental questionnaires were administered during pregnancy, at or shortly after birth, and again at regular intervals until children were 11 years, 10 years or 9 years of age in PIAMA, LISAplus and INMA, respectively. In this investigation, we focused on the following later childhood health outcomes: current asthma at 6 years of age; current asthma at 10 years of age; and doctor-diagnosed asthma ever assessed at 10 years of age. Current asthma at 6 years or 10 years of age was defined as fulfilling at least two out of three parent-reported conditions: 1) doctor-diagnosed asthma ever; 2) reported wheezing symptoms in the past 12 months, based on the International Study of Asthma and Allergy in Childhood core questions [20]; and 3) reported asthma medication intake/asthma treatment in the past 12 months [21, 22]. Controls were defined as "complete" controls and had information available for all time-points. Specific IgE to aero-allergens, assessed at 8 years and 10 years of age, were available for PIAMA and LISAplus, respectively. Blood samples were analysed for total and specific IgE. Allergic sensitisation was defined as positive if the specific IgE was $\geqslant 0.35 \mathrm{kU} \cdot \mathrm{L}^{-1}$. In LISAplus, the "sx1 inhalant mixture" (timothy, rye, mugwort, mite, cat, dog and mould mixture) was used to assess aero-allergen sensitisation. In PIAMA, aero-allergen sensitisation was assessed against house dust mite (Der p), cat, dog, birch, Alternaria Alternata or Dactylis glomerata allergens. For stratification analyses, atopic asthma ever at 10 years of age was defined as positive when a child had doctor-diagnosed asthma ever at 10 years of age and was sensitised to aero-allergens at 10 years of age. Non-atopic asthma ever at 10 years of age was defined as having a diagnosis of asthma ever at 10 years of age, but without concomitant aero-allergen sensitisation. Controls were identified as those never reporting doctor-diagnosed asthma up to 10 years of age and not being sensitised to aero-allergens at 10 years of age. Information on atopic and non-atopic asthma ever was only available for the PIAMA and LISAplus birth cohorts.

\section{Statistical analysis}

Concentrations of endotoxins, (1,3)- $\beta$-D-glucan and EPS were naturally log-transformed. Correlations between all bio-contaminant concentrations were assessed using the Pearson product-moment correlation coefficient (r). Logistic regression was used to analyse the associations between exposure to bio-contaminants and health outcomes. Associations are presented per interquartile range increase in bio-contaminant concentrations and loads (log-transformed) in dust from living room floors (PIAMA and LISAplus) and sofas (INMA). Generalised additive models (GAM) were used to assess relationships between exposure to bio-contaminants in dust and health outcomes (R project; http://cran.r-project.org/ web/packages/gam/index.html). For nonlinear relationships, the bio-contaminants were categorised into tertiles and analysed using logistic regression. A common set of confounding factors was defined in all cohorts. Confounders were selected based on association analyses in relation to the health outcomes, as well as based on the previous literature. All models were adjusted for sex, maternal smoking during pregnancy, parental asthma, dog ownership (at 0-1 years of age), cat ownership (at 0-1 years of age), mould in the home (at 3 months of age), breastfeeding, parental education level, and season of dust sampling. In addition, stratified analyses were performed for atopic asthma ever at 10 years of age and non-atopic asthma ever at 10 years of age in PIAMA and LISAplus. The results are presented as odds ratios with corresponding $95 \%$ confidence intervals for the total study population and separately by birth cohort due to the data heterogeneity.

Associations between health outcomes and exposure to bio-contaminants, expressed as loads (EU.m ${ }^{-2}$ and EPSU $\cdot \mathrm{m}^{-2}$ ), were assessed in the PIAMA and INMA cohorts only, as this information was not sufficiently available in LISAplus. Since information on $(1,3)-\beta$-D-glucan loads was only available in PIAMA, we only present the results for endotoxin and EPS. All statistical analyses were performed using the statistical software R, version 2.14.1 (www.R-project.org). 


\section{Results}

Study characteristics and health information

In total, 1429 children predominantly living in (sub)-urban areas were included in the analysis (PIAMA: $\mathrm{n}=553$; INMA: $\mathrm{n}=481$; and LISAplus: $\mathrm{n}=395$ ) (table 1). INMA participants were more frequently exposed to maternal smoking during pregnancy (38\% versus $13 \%$ in PIAMA and $14 \%$ in LISAplus). Parental asthma was more prevalent in the PIAMA cohort compared to the other cohorts $(33 \%$ versus $11 \%$ in INMA and $12 \%$ in LISAplus). Parents of the PIAMA and LISAplus participants were more likely to report completing higher education (54\% and $80 \%$, respectively) compared to the parents of the INMA participants (17\%). Breastfeeding during the first 6 months of life was common, with a prevalence of $>82 \%$ among all birth cohort samples. Because the HITEA study includes subsets of the PIAMA and LISAplus population, Chi-squared tests were performed to compare the sociodemographic characteristics presented in table 1, between children who were and were not included in the current analysis. Parents of the PIAMA children included in the HITEA study reported significantly less maternal smoking during pregnancy (13\% versus $18 \%$ ) and a higher prevalence of parental asthma (33\% versus $15 \%$ ) compared to those not included, respectively. LISAplus children in HITEA were more frequently born to parents with a high education level ( $80 \%$ versus $64 \%$ ) and to mothers who did not smoke during pregnancy (14\% versus $17 \%)$ compared to the LISAplus children who did not participate in HITEA, respectively.

Half of the children participating in INMA (52\%) were exposed to visible mould in the home at birth, compared to $40 \%$ in PIAMA and 25\% in LISAplus. Dog ownership in the first year of life was more often reported among the Spanish children (35\%), whereas cat ownership was most prevalent among Dutch children (26\%). More than two-thirds (78\%) of the children participating in INMA shared their bedroom, compared to only $7 \%$ of the PIAMA participants. No information was available for this covariate in LISAplus. Day care attendance in the first 2 years of life was common among both Dutch and Spanish participants (67\% and $85 \%$, respectively), but only $6 \%$ of German parents reported that their child attended day care. As there was no evidence for an association between day care attendance and all investigated health outcomes in the birth cohorts (Chi-squared test $\mathrm{p}<0.1$ ), day care attendance was not included as a possible confounder in the adjusted logistic regression analyses.

Current asthma at 6 years (7\%) and 10 years of age (6\%) and ever being diagnosed with asthma at 10 years of age (16\%) was more prevalent among Dutch children, compared to the Spanish and German

\section{TABLE 1 Characteristics of the study population}

\begin{tabular}{|c|c|c|c|c|}
\hline Characteristics & Total & PIAMA & INMA & LISAplus \\
\hline Subjects $\mathrm{n}$ & 1492 & 553 & 481 & 395 \\
\hline \multicolumn{5}{|l|}{ Sociodemographics } \\
\hline Maternal smoking during pregnancy & $308 / 1398(22)$ & $72 / 540(13)$ & $182 / 481$ (38) & $54 / 377(14)$ \\
\hline Parental asthma & $274 / 1397(20)$ & $177 / 542(33)$ & $51 / 475(11)$ & $46 / 380(12)$ \\
\hline High parental education ${ }^{\#}$ & $657 / 1344(49)$ & $271 / 503(54)$ & $79 / 459(17)$ & $307 / 382(80)$ \\
\hline Mould at home at birth & $544 / 1360(40)$ & $196 / 492(40)$ & $248 / 473(52)$ & $100 / 395(25)$ \\
\hline Dog ownership at $0-1$ years of age & $264 / 1095$ (19) & $76 / 510(15)$ & $166 / 477(35)$ & $22 / 372(6)$ \\
\hline Cat ownership at $0-1$ years of age & $241 / 1365$ (18) & $132 / 511(26)$ & $69 / 478(14)$ & $40 / 376(11)$ \\
\hline Sharing a bedroom at $0-1$ years of age & $400 / 890(45)$ & $27 / 411(7)$ & $373 / 479(78)$ & \\
\hline Attending day care at $1-2$ years of age & $749 / 1329(56)$ & $326 / 489(67)$ & $400 / 468(85)$ & $23 / 372(6)$ \\
\hline \multicolumn{5}{|l|}{ Health outcomes } \\
\hline
\end{tabular}

Data are presented as $\mathrm{n} / \mathrm{N}(\%)$, unless otherwise stated. PIAMA: Prevention and Incidence of Asthma and Mite Allergy; INMA: INfancia y Medio Ambiente - Childhood and Environment; LISAplus: Influence of Life-style factors on the development of the Immune System and Allergies in East and West Germany. ": highest education level (country-specific education system), either father or mother; ": exclusive breastfeeding or breastfeeding and bottle feeding during the first 6 months of life; ${ }^{+}$: exact age at follow-up was 11,9 and 10 years of age in PIAMA, INMA and LISAplus, respectively; ${ }^{\S}$ : at 8 years of age in PIAMA: specific $\operatorname{lgE}$ to at least one allergen $\left(\geqslant 0.35 \mathrm{kU} \cdot \mathrm{L}^{-1}\right) \mathrm{mite}$, cat, dog, birch, Alternaria alternate or Dactylis glomerata (grass); at 10 years of age in LISAplus: specific $\lg E\left(\geqslant 0.35 \mathrm{kU} \cdot \mathrm{L}^{-1}\right)$ to sx1 inhalant mixture (timothy, rye, mugwort, mite, car, dog or mould mixture). 
children (3\%, $4 \%$ and $6 \%$, respectively, for both cohorts). At 8 years of age, 39\% of the PIAMA cohort was sensitised to aero-allergens, compared to $22 \%$ of the LISAplus cohort at 10 years of age.

\section{Distribution of the bio-contaminants in settled dust}

The distribution of the bio-contaminant levels is presented in table 2. A detailed overview can be found elsewhere [19]. Briefly, the median endotoxin concentration was lowest in INMA (3.62 EU.mg ${ }^{-1}$ ), compared to $23.87 \mathrm{EU} \cdot \mathrm{mg}^{-1}$ and $21.75 \mathrm{EU} \cdot \mathrm{mg}^{-1}$ in PIAMA and LISAplus, respectively. In contrast, the median concentration of fungal EPS was highest in INMA $\left(109.60 \mathrm{EPSU} \cdot \mathrm{mg}^{-1}\right.$ versus $29.68 \mathrm{EPSU} \cdot \mathrm{mg}^{-1}$ and 45.36 EPSU $\cdot \mathrm{mg}^{-1}$ in PIAMA and LISAplus, respectively). No major differences were observed in $(1,3)$ - $\beta$-D-glucan concentrations between the Dutch and German cohorts. When expressed as total burden per sampled $\mathrm{m}^{2}$ (loads), the difference in EPS levels between PIAMA and INMA was reduced.

\section{Correlation between bio-contaminants}

In general, there was no correlation between endotoxin and (1,3)- $\beta$-D-glucan concentrations (PIAMA: Pearson's $r=-0.04$; LISAplus Pearson's $r=0.001$ ). A low, significant correlation between endotoxin and EPS was observed for LISAplus $(\mathrm{r}=0.19, \mathrm{p}<0.001)$, but not for PIAMA $(\mathrm{r}=-0.09)$ or INMA $(\mathrm{r}=-0-05)$. The correlation between endotoxin and EPS loads in settled dust from the living room was low (INMA, $\mathrm{r}$ : $0.10, \mathrm{p}<0.05)$ to moderate (PIAMA, $\mathrm{r}=0.33, \mathrm{p}<0.001$ ). We also examined whether there was an association between parental-reported mould in the home shortly after birth and the levels of (1,3)- $\beta$-D-glucan and EPS in the living room floor dust (Wilcoxon test) in all three birth cohorts. For INMA, EPS concentrations in the living room floor dust were significantly higher in homes with reported mould problems compared to homes with no reported mould problems (data not shown). As parental-reported mould in the home around the time of birth was not associated with any of the health outcomes tested, this variable was not included as a possible confounder within the adjusted analyses (data not shown).

Early exposure to bio-contaminants in relation to asthma and specific IgE to aero-allergens

The results of the adjusted logistic regression analyses, stratified by birth cohort, are presented in table 3 . In PIAMA, endotoxin concentrations in living room floor dust were significantly associated with a higher prevalence of current asthma at 6 years of age (adjusted OR 1.96, 95\% CI 1.07-3.58), but not at 10 years of age or ever up to the age of 10 years. In LISAplus, no associations were found between endotoxin levels and the asthma outcomes at any time-point. In the INMA cohort, there was a significant inverse

TABLE 2 Distribution of the bio-contaminants (original scale)

\begin{tabular}{|c|c|c|c|c|c|c|}
\hline & $\mathbf{N}$ & $1^{\text {st }}$ quartile & Median & Geometric mean & $3^{\text {rd }}$ quartile & IQR \\
\hline \multicolumn{7}{|c|}{ Endotoxin EU.mg ${ }^{-1}$} \\
\hline Total & 1383 & 5.08 & 15.76 & 11.76 & 35.05 & 29.97 \\
\hline INMA & 460 & 1.04 & 3.62 & 3.27 & 11.50 & 10.46 \\
\hline LISAplus & 394 & 13.49 & 21.75 & 22.77 & 36.72 & 23.23 \\
\hline \multicolumn{7}{|c|}{$(1,3)-\beta$-D-glucan $\mu \mathrm{g} \cdot \mathrm{mg}^{-1}$} \\
\hline LISAplus & 395 & 1.47 & 2.00 & 1.91 & 2.55 & 1.08 \\
\hline \multicolumn{7}{|c|}{ Extracellular polysaccharides EPSU $\cdot \mathrm{mg}^{-1}$} \\
\hline Total & 1362 & 26.37 & 53.36 & 53.61 & 108.00 & 81.67 \\
\hline PIAMA & 514 & 14.64 & 29.68 & 30.03 & 56.67 & 42.03 \\
\hline INMA & 453 & 66.02 & 109.60 & 115.07 & 184.10 & 118.10 \\
\hline LISAplus & 395 & 27.86 & 45.36 & 47.46 & 78.96 & 51.10 \\
\hline \multicolumn{7}{|c|}{ Extracellular polysaccharides ${ }^{\#}$ EPSU $\cdot \mathrm{m}^{-2}$} \\
\hline Total & 970 & 1.51 & 7.25 & 7.37 & 28.12 & 30.36 \\
\hline PIAMA & 515 & 0.61 & 8.37 & 6.00 & 28.65 & 28.04 \\
\hline INMA & 455 & 2.61 & 8.55 & 9.29 & 35.90 & 33.28 \\
\hline
\end{tabular}


TABLE 3 Association between exposure to measured bio-contaminants with asthma outcomes and specific IgE to aero-allergens, stratified by cohort

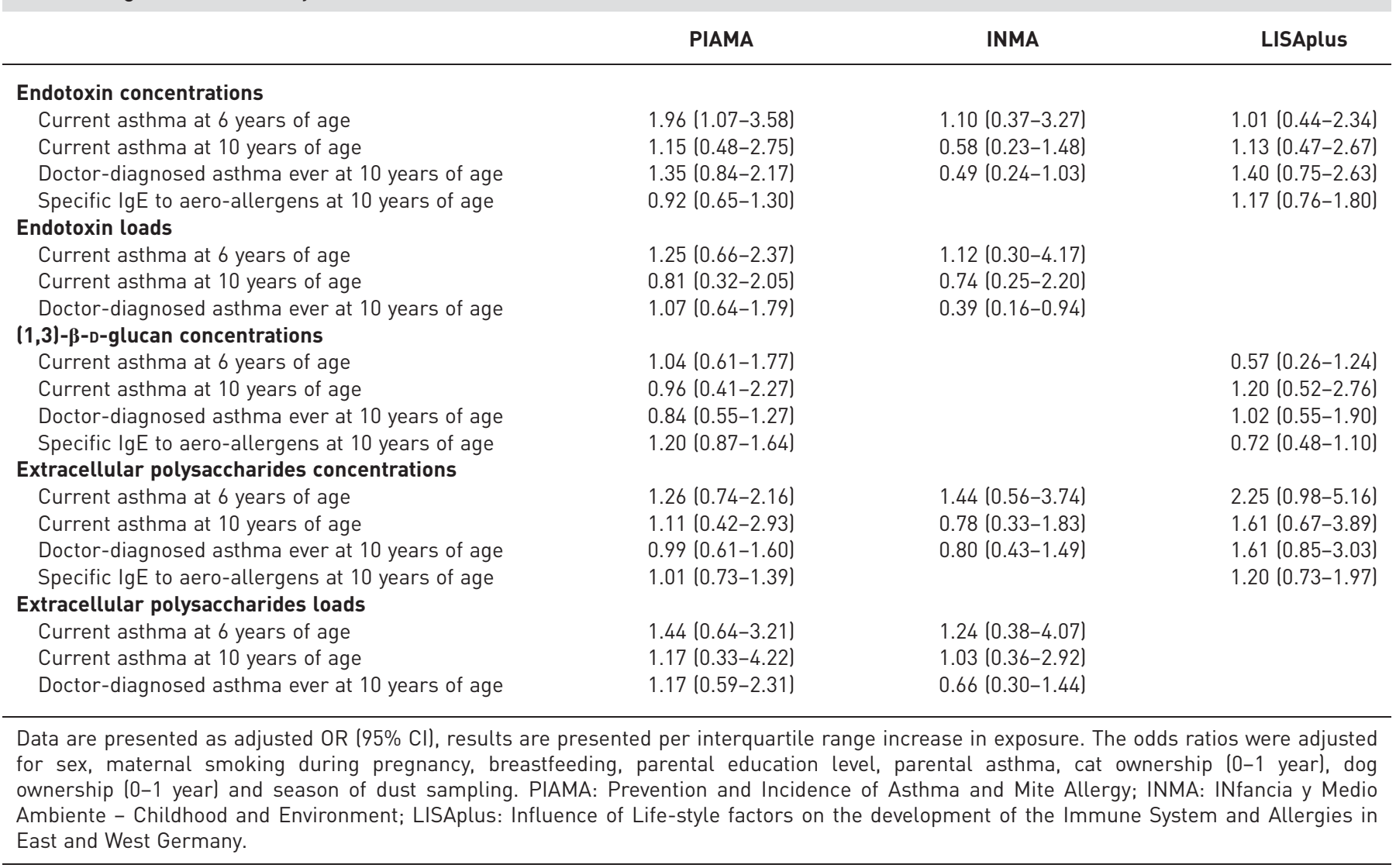

association between endotoxin loads and doctor-diagnosed asthma ever up to 10 years of age (adjusted OR 0.39, 95\% CI 0.16-0.94). No significant associations were found between (1,3)- $\beta$-D-glucan and the health outcomes in the PIAMA and LISAplus samples. There was a (nonsignificant) increased risk of current asthma at 6 years of age, which was associated with exposure to high levels of fungal EPS in all participating cohorts. For the asthma outcomes at 10 years of age, the strength of these associations was diminished. Exposure to EPS expressed as loads was positively associated with asthma at 6 years and 10 years of age within the PIAMA cohort. Exposure to EPS was inversely, but not significantly, associated with lifetime prevalence of asthma at 10 years of age in INMA, as was observed for endotoxin in this cohort. No association was found between any of the measured exposures and aero-allergen sensitisation in later childhood.

In analyses stratified by atopy at 10 years of age, we found no statistical association between early exposure to bio-contaminants in living room floor dust and children who had received a physician diagnosis of asthma by 10 years of age, with or without an atopic condition. Although for PIAMA inverse associations were observed in the entire subsample, there were no major differences in associations between atopic and non-atopic children. Unfortunately, it was not possible to investigate associations between exposure to bio-contaminants in relation to asthma among non-atopic children in LISAplus due to the low subject numbers.

\section{Exposure categorised into tertiles}

We assessed the linearity of the relationships between bio-contaminant exposures and health outcomes using GAM. The GAM plots (fig. S1a-f) indicated that some of the investigated relationships deviate from linearity. In these cases, adjusted logistic regression analyses were performed after grouping the exposure into tertiles. However, none of the investigated associations were statistically significant (fig. S1a-f).

\section{Discussion}

We prospectively investigated whether early-life exposure to endotoxins, (1,3)- $\beta$-D-glucan and EPS, measured in settled dust from living rooms, plays a role in asthma and atopy development in children up to 10 years of age using data from three European birth cohorts. The birth cohort samples comprised 
children living in predominantly (sub)-urban areas. The results of our analysis are inconsistent; both positive and negative associations were observed. To the best of our knowledge, the present study is among only a few that have prospectively investigated associations between early exposure to bio-contaminants and respiratory and allergic health outcomes during later childhood, using data which covers different geographical regions in Europe.

Currently, the long-term effects of early exposure to bio-contaminants in relation to health and allergy development at later ages are under investigated, especially for populations living in (sub)-urban environments. Only a few studies can be considered as comparable to the current study in terms of design and exposure definition. A birth cohort study from the USA, conducted in the metropolitan area of Boston, measured endotoxin levels in dust taken from sofas and the surrounding floor in the family room at 3 months of age. No significant associations were seen with recurrent wheeze at 3 years of age [23], but endotoxin levels were positively associated with persistent wheeze at 7 years of age (adjusted OR 3.50, 95\% CI 1.30-9.80). However, there was a decreased risk of sensitisation to at least one allergen (IgE/skin prick test) in relation to moderate endotoxin concentrations at 7 years of age [11]. In a recent HITEA investigation based on the same study subjects included in the current analysis, exposure to endotoxin shortly after birth was significantly inversely associated with exhaled nitric oxide at 10 years of age [12]. Furthermore, in the same subset of the PIAMA cohort as used in the current study, DouwEs et al. [10] reported that exposure to high amounts of endotoxins (loads) at 3 months of age was inversely associated with ever having doctor-diagnosed asthma up to 4 years of age, and furthermore, that high exposure to EPS (loads) was also associated with a decreased risk of persistent wheeze up to 4 years of age. A few years later, within the HITEA study, positive associations were observed at 6 years and 10 years of age among the children from PIAMA, which contradicts these previous findings. One explanation for this discrepancy may be related to the fact that the definition of ever asthma outcome in the present study includes asthma cases diagnosed up to a much older age. In addition, respiratory infections have been associated with wheezing illnesses and asthma in children [24], and they might have the potential to influence the pathophysiology of asthma, especially among younger school-aged children [25]. Therefore, we tested whether the association between early exposure to bio-contaminants and doctor-diagnosed asthma at 6 years of age was affected by concomitant bronchitis. Information on current bronchitis was available in PIAMA and LISAplus only. The effect estimates did not change substantially after adjusting for current bronchitis, which might indicate that current bronchitis at 6 years of age does not influence the biological pathway between exposure to bio-contaminants and current asthma at 6 years of age in LISAplus and PIAMA (data not shown). However, we are unable to assess and replicate this result in the INMA cohort because data are unavailable. Furthermore, the association between exposure to bio-contaminants might be influenced by atopic status, as suggested by BRAUN-FAHRLÄNDER et al. [6]. Therefore, we stratified the analyses by atopic status at 10 years of age, However, no statistically significant associations were observed and there were no major differences in the results between the two strata with regards to doctor-diagnosed asthma ever at 10 years of age (data not shown).

In the frame of the so-called "farm studies", the PARSIFAL (Prevention of Allergy - Risk Factors for Sensitization Related to Farming and Anthroposophic Lifestyle) study, a cross-sectional study of children aged 5-13 years recruited among farmers, families with an anthroposophic lifestyle and their respective reference groups in five European countries, found no effect of exposure to bio-contaminants on current atopic wheeze in farm children [3]. Additionally, there was no evidence of an association among children from families with an anthroposophic lifestyle (Steiner children) and their respective reference group (from (sub)-urban environments). Moreover, exposure to endotoxin was (not significantly) associated with an increased risk for current atopic wheeze in Steiner children in mutually adjusted analyses. The findings reported for Steiner children might be comparable to what has been found in our study for current asthma at 6 years of age. However, comparisons between the PARSIFAL and HITEA studies should be done with caution as they have different study designs (cross-sectional versus early exposure and later health outcomes) and the HITEA study has an emphasis on mainly urban settings.

Within the HITEA study, we observed that the distribution of the bio-contaminants differed among the three birth cohorts, especially for endotoxin and EPS. This might either reflect true differences among the three studies or might partly be a result of different sampling and analytical procedures. Unlike for PIAMA and LISAplus, INMA dust samples were taken from the sofa in the living room. The different sampling locations could reflect different sources of microbial exposure, resulting in diverse exposure levels [26]. A study by Thorne et al. [27] on predictors of household endotoxin compared five sampling locations within the home. They observed that endotoxin concentrations from family room floor dust were lower than endotoxin concentrations sampled from the family room sofa; however, the endotoxin loads were comparable [27]. This could indicate that the total amount of dust or unknown, not measured viable and non-viable substances might play a more decisive role. Unfortunately, we were not able to compare 
the total amount of dust among the three birth cohorts as this information was only available for PIAMA. In addition, the INMA samples were extracted in borate buffered saline for previous mite allergen analyses, which is not the standard extraction fluid used for endotoxin analyses, and thus might have led to lower levels for INMA [19]. These methodological differences might in turn be responsible for the heterogeneity of the effect estimates between the cohorts, but are probably not crucial as analyses were conducted per cohort specific interquartile range increase in exposure.

Several factors may play a role in the relationship between early exposure to bio-contaminants and health outcomes in later childhood. For children living on farms, it was recently suggested that a higher and more diverse microbial environment indoors might have stronger and more persistent immunological effects than simply the quantity of specific bio-contaminant exposures [28, 29]. Unfortunately, for this investigation, we were not able to describe the microbial exposure in any greater detail, e.g. by defining microbial diversity [30]. However, important previous key publications in farming and non-farming environments have investigated the effect of exposure to bacterial endotoxin, and maintaining comparability to other studies is essential in order to validate and evaluate our findings.

Although the present study has important strengths, such as the inclusion of different geographical regions and a similar, prospective study design, some limitations should be noted. For the present HITEA investigation, only sub-samples of the original LISAplus and PIAMA birth cohorts were included in the HITEA study. In PIAMA, only children from the intervention arm of the study were included in the HITEA study. These are children from allergic and/or asthmatic mothers, which might explain the higher asthma lifetime prevalence in this cohort compared to the other two [31]. Although a previous HITEA study reported that there are no differences in any of the determinants that are significantly associated with microbial agents between those participating in HITEA and the whole cohorts [19], the HITEA population differed significantly from those not included with regards to sociodemographic characteristics, such as maternal smoking during pregnancy (PIAMA and LISAplus), parental asthma (PIAMA) and parental education level (LISAplus). We assume that these differences are unlikely to affect the direction and the magnitude of the effects dramatically, but we are unable to confirm this assumption. Furthermore, although we have information up to 11 years of age, we were not able to investigate incident asthma after 6 years of age due to the limited numbers of incident asthma cases. Therefore, we cannot determine whether the effect of early exposure to bio-contaminants and respiratory outcomes continues to progress even after 6 years of age. Finally, the Institute of Medicine (Washington D.C. USA) suggested in 2004 that sampled floor dust concentrations per $\mathrm{m}^{2}$ might be more accurate for exposure assessment purposes than concentrations per gram of sampled dust [32]. Unfortunately, for LISAplus, exposure information was only available as floor dust concentrations per gram of sampled dust. However, according to table 3, this does not appear to have had a considerable impact on the direction of the investigated exposure response relationships in PIAMA and INMA. There were only slight changes in the effect estimates for the associations between exposure to endotoxin and current asthma at 10 years of age and exposure to EPS and doctor-diagnosed asthma ever up to 10 years of age within PIAMA, when comparing the two different exposure metrics.

Little is known regarding whether a single bio-contaminant measurement is able to represent the overall exposure within a home, as the microbial components in house dust samples may change over time $[30,33]$. We chose to focus particularly on early exposure shortly after birth. Whereas PARK et al. [13] observed that the correlation for repeated endotoxin measurements over time was higher for bed than floor dust, a single measurement of endotoxin has been observed to be representative for at least a 1-year period within the INGA (Indoor Factors and Genetics and Asthma) study [34]. To the best of our knowledge, we are not aware of similar studies regarding temporal changes of $(1,3)$ - $\beta$-D-glucan or EPS exposure within homes. Nevertheless, we cannot exclude the possibility that the suggested immunomodulatory effects of bio-contaminant exposure on respiratory health may not only occur shortly after birth, but rather that a constant signal in later childhood is also necessary. A US birth cohort study in the Boston area demonstrated a borderline independent inverse effect of concurrent exposure to endotoxin on current asthma in 7-year-old children [35], but this effect was only significant when endotoxin exposure was high at both birth and 7 years of age. Moreover, with increasing age, the school environment and activities conducted in different places might start to become more important and the daily individual microbial exposure may change in composition [36-38]. One study in the USA demonstrated that school-aged children with asthma were exposed to higher concentrations of endotoxin in their classrooms than in their home environments [38]. In the Netherlands, higher levels of endotoxins have been reported in classrooms compared to residences as well, and these former levels were shown to be associated with an increased risk of non-atopic asthma after mutual adjustment for home endotoxin exposure levels [37].

The results of the current study conducted in children from (sub)-urban areas does not provide a definite conclusion regarding the impact of early exposure to bio-contaminants in settled dust from living rooms 
on the development of asthma and atopy. Our data are not in line with previous findings from cross-sectional studies conducted predominantly in rural areas or from prospective studies on younger children. Our findings indicate that the suggested immunological mechanisms hypothesised to link exposure to a rich microbial environment in early life with asthma and allergy, as observed in children from farms and rural environments, might either be weaker or not true for children growing up in (sub)-urban environments, or might not affect health outcomes in later childhood. With increasing age, exposures that occur outside of the home, such as in the school environment, might be of increasing importance. These additional exposures remain largely under investigated. Only a few birth cohort studies set in farming, rural and (sub)-urban environments have the potential to look at the effects of early exposure to microbial agents on health outcomes in later childhood.

\section{References}

1 Prescott SL, Macaubas C, Smallacombe T, et al. Development of allergen-specific T-cell memory in atopic and normal children. Lancet 1999; 353: 196-200.

2 Von Mutius E. Epidemiology of allergic diseases. In: Leung DY, Sampsom, HA, Geha R, et al., eds. Pediatric Allergy: Principles and Practice. St Louis, Mosby, 2010; pp. 1-8.

3 Schram-Bijkerk D, Doekes G, Douwes J, et al. Bacterial and fungal agents in house dust and wheeze in children: the PARSIFAL study. Clin Exp Allergy 2005; 35: 1272-1278.

4 van Strien RT, Engel R, Holst O, et al. Microbial exposure of rural school children, as assessed by levels of $\mathrm{N}$-acetyl-muramic acid in mattress dust, and its association with respiratory health. J Allergy Clin Immunol 2004; 113: 860-867.

5 Karvonen AM, Hyvarinen A, Gehring U, et al. Exposure to microbial agents in house dust and wheezing, atopic dermatitis and atopic sensitization in early childhood: a birth cohort study in rural areas. Clin Exp Allergy 2012; 42: $1246-1256$

6 Braun-Fahrlander C, Riedler J, Herz U, et al. Environmental exposure to endotoxin and its relation to asthma in school-age children. N Engl J Med 2002; 347: 869-877.

7 Eduard W, Omenaas E, Bakke PS, et al. Atopic and non-atopic asthma in a farming and a general population. Am Ind Med 2004; 46: 396-399.

8 Gehring U, Bolte G, Borte M, et al. Exposure to endotoxin decreases the risk of atopic eczema in infancy: a cohort study. J Allergy Clin Immunol 2001; 108: 847-854.

9 Iossifova YY, Reponen T, Bernstein DI, et al. House dust (1-3)- $\beta$-D-glucan and wheezing in infants. Allergy 2007; 62: 504-513.

10 Douwes J, van Strien R, Doekes G, et al. Does early indoor microbial exposure reduce the risk of asthma? The Prevention and Incidence of Asthma and Mite Allergy birth cohort study. J Allergy Clin Immunol 2006; 117: $1067-1073$

11 Celedon JC, Milton DK, Ramsey CD, et al. Exposure to dust mite allergen and endotoxin in early life and asthma and atopy in childhood. J Allergy Clin Immunol 2007; 120: 144-149.

12 Casas L, Tischer C, Wouters IM, et al. Early life microbial exposure and fractional exhaled nitric oxide in school-age children: a prospective birth cohort study. Environ Health 2013; 12: 103.

13 Park JH, Gold DR, Spiegelman DL, et al. House dust endotoxin and wheeze in the first year of life. Am J Respir Crit Care Med 2001; 163: 322-328.

14 Litonjua AA, Milton DK, Celedon JC, et al. A longitudinal analysis of wheezing in young children: the independent effects of early life exposure to house dust endotoxin, allergens, and pets. J Allergy Clin Immunol 2002; 110: 736-742.

15 Bolte G, Bischof W, Borte M, et al. Early endotoxin exposure and atopy development in infants: results of a birth cohort study. Clin Exp Allergy 2003; 33: 770-776.

16 Torrent M, Sunyer J, Garcia R, et al. Early-life allergen exposure and atopy, asthma, and wheeze up to 6 years of age. Am J Respir Crit Care Med 2007; 176: 446-453.

17 Wijga $\mathrm{AH}$, Kerkhof $\mathrm{M}$, Gehring $\mathrm{U}$, et al. Cohort profile: the Prevention and Incidence of Asthma and Mite Allergy (PIAMA) birth cohort. Int J Epidemiol 2014; 43: 527-535.

18 Heinrich J, Bolte G, Hölscher B, et al. Allergens and endotoxin on mothers' mattresses and total immunoglobulin $\mathrm{E}$ in cord blood of neonates. Eur Respir J 2002; 20: 617-623.

19 Casas L, Tischer C, Wouters IM, et al. Endotoxin, extracellular polysaccharides, and $\beta(1-3)$-glucan concentrations in dust and their determinants in four European birth cohorts: results from the HITEA project. Indoor Air 2013; 23: 208-218.

20 Asher MI, Keil U, Anderson HR, et al. International Study of Asthma and Allergies in Childhood (ISAAC): rationale and methods. Eur Respir J 1995; 8: 483-491.

21 Lodrup Carlsen KC, Haland G, Devulapalli CS, et al. Asthma in every fifth child in Oslo, Norway: a 10-year follow up of a birth cohort study. Allergy 2006; 61: 454-460.

22 Lodrup Carlsen KC, Roll S, Carlsen KH, et al. Does pet ownership in infancy lead to asthma or allergy at school age? Pooled analysis of individual participant data from 11 European birth cohorts. PLoS One 2012; 7: e43214.

23 Ryan PH, Bernstein DI, Lockey J, et al. Exposure to traffic-related particles and endotoxin during infancy is associated with wheezing at age 3 years. Am J Respir Crit Care Med 2009; 180: 1068-1075.

24 Gern JE. Viral respiratory infection and the link to asthma. Pediatr Infect Dis J 2008; 27: Suppl. 10, S97-S103.

25 Guilbert TW, Gern, JE , Lemanske RF. Infections and asthma. In: Leung DY, Sampsom, HA, Geha R, et al., eds. Pediatric Allergy: Principles and Practice. St Louis, Mosby, 2010; pp. 363-376.

26 Frankel M, Timm M, Hansen EW, et al. Comparison of sampling methods for the assessment of indoor microbial exposure. Indoor Air 2012; 22: 405-414.

27 Thorne PS, Cohn RD, Mav D, et al. Predictors of endotoxin levels in U.S. housing. Environ Health Perspect 2009; 117: 763-771. 
28 Ege MJ, Mayer M, Normand AC, et al. Exposure to environmental microorganisms and childhood asthma. $N$ Engl J Med 2011; 364: 701-709.

29 Ege MJ, Mayer M, Schwaiger K, et al. Environmental bacteria and childhood asthma. Allergy 2012; 67: 1565-1571.

30 Tischer CG, Heinrich J. Exposure assessment of residential mould, fungi and microbial components in relation to children's health: achievements and challenges. Int J Hyg Environ Health 2013; 216: 109-114.

31 Caudri D, Savenije OE, Smit HA, et al. Perinatal risk factors for wheezing phenotypes in the first 8 years of life. Clin Exp Allergy 2013; 43: 1395-1405.

32 Institue ofMedicine . Damp Indoor Spaces and Health. Washington, D.C., National Academy Press, 2004.

33 Tischer C, Gehring U, Chen CM, et al. Respiratory health in children, and indoor exposure to (1,3)- $\beta$-D-glucan, EPS mould components and endotoxin. Eur Respir J 2011; 37: 1050-1059.

34 Heinrich J, Holscher B, Douwes J, et al. Reproducibility of allergen, endotoxin and fungi measurements in the indoor environment. J Expo Anal Environ Epidemiol 2003; 13: 152-160.

35 Sordillo JE, Hoffman EB, Celedon JC, et al. Multiple microbial exposures in the home may protect against asthma or allergy in childhood. Clin Exp Allergy 2010; 40: 902-910.

36 Jacobs JH, Krop EJ, Borras-Santos A, et al. Endotoxin levels in settled airborne dust in European schools: the HITEA school study. Indoor Air 2014; 24: 148-157.

37 Jacobs JH, Krop EJ, de Wind S, et al. Endotoxin levels in homes and classrooms of Dutch school children and respiratory health. Eur Respir J 2013; 42: 314-322.

38 Sheehan WJ, Hoffman EB, Fu C, et al. Endotoxin exposure in inner-city schools and homes of children with asthma. Ann Allergy Asthma Immunol 2012; 108: 418-422. 